ACR damage index (SDI) and HR-QoL by EQ-5D were measured. Bivariate analysis through chi squared and U Mann Whitney Multivariate analysis was performed by logistic regression to adjust for significant associations. Statistical analysis for related samples was evaluated with Mc. Nemar test.

Results We analyzed 400 Colombian patients. Baseline median age was 49 years (15 IQR) with median age at diagnosis and disease duration of 37 years (17 IQR) and 9 years (13 IQR) respectively. There were $94 \%$ female patients and $17.3 \%$ late onset SLE. Most frequent clinical manifestations were hematological (82.8\%), mucocutaneous (75.3\%) and nephritis (33.8\%). Only $4.5 \%$ had neurological involvement. The mean SLEDAI were 1.18 and 0.65 at first and second measurement respectively, in the first measurement $97.1 \%$ of the patients had a SLEDAI $\leq 4$. The mean SDI was 0.7275 at first measurement and 0.985 at the second measurement. Although SDI was associated to various dimensions of HR-QoL measured by ED-5D, disease activity was not related (See table 1).

Conclusions In SLE Colombian patients with a stablished disease and an altered HR-QoL, low disease activity is not related with HR-QoL when measured by EQ-5d. In the present study, it is highlighted that while disease activity decreases, damage increases. Damage accrual has a relation with HR-QoL in the short term. The impact and correlation must be better defined in a long-term follow-up. The associated effect on HRQOL emphasizes the need for strategies to reduce the risk of cumulative organ damage.

\section{CS-36 RECOMMENDATIONS FOR THE ASSESSMENT OF SYSTEMIC LUPUS ERYTHEMATOSUS IN CANADA}

${ }^{1}$ Stephanie Keeling*, ${ }^{2}$ Zainab Alabdurubalnabi, ${ }^{2}$ Antonio Avina-Zubieta, ${ }^{3}$ Susan Barr, ${ }^{4}$ Louise Bergeron, ${ }^{5}$ Sasha Bernatsky, ${ }^{6}$ Josiane Bourre-Tessier, ${ }^{3}$ Ann Clarke, ${ }^{6}$ Alexandra BarilDionne, ${ }^{2}$ Jan Dutz, ${ }^{2}$ Stephanie Ensworth, ${ }^{3}$ Aurore Fifi-Mah, ${ }^{7}$ Paul R Fortin, ${ }^{8}$ Dafna Gladman, ${ }^{9}$ Derek Haaland, ${ }^{10}$ John G Hanly, ${ }^{11}$ Linda T Hiraki, ${ }^{6}$ Sara Hussein, ${ }^{9}$ Kimberly Legault, ${ }^{11}$ Deborah Levy, ${ }^{12}$ Lily Lim, ${ }^{9}$ Mark Matsos, ${ }^{13}$ Emily G McDonald, ${ }^{8}$ Jorge Medina-Rosas, ${ }^{14}$ Jordi Pardo Pardi, ${ }^{12}$ Christine Peschken, ${ }^{5}$ Christian Pineau, ${ }^{15}$ Janet Pope, ${ }^{16}$ Tamara Rader, ${ }^{2}$ Jen Reynolds, ${ }^{11}$ Earl Silverman, ${ }^{8}$ Konstantinos Tselios, ${ }^{6}$ Manon Suitner, ${ }^{8}$ Murray Urowitz, ${ }^{8}$ Zahi Touma, ${ }^{5}$ Evelyne Vinet, ${ }^{17}$ Nancy Santesso. ${ }^{1}$ Divison of Rheumatology, Department of Medicine, University of Alberta; ${ }^{2}$ Division of Rheumatology, Department of Medicine, University of British Columbia; ${ }^{3}$ Division of Rheumatology, Department of Medicine, Cumming School of Medicine, University of Calgary; ${ }^{4}$ Louise Bergeron, Canadian Arthritis Patient Alliance; ${ }^{5}$ Division of Rheumatology, Department of Medicine, McGill University; ${ }^{6}$ Division of Rheumatology, Department of Medicine, Université de Montreal; ${ }^{7}$ Division of Rheumatology, Department of Medicine, Université Laval; ${ }^{8}$ Institute of Health Policy, Management and Evaluation, University of Toronto; ${ }^{9}$ Division of Rheumatology, Department of Medicine, McMaster University; ${ }^{10}$ Division of Rheumatology, Department of Medicine, Dalhousie University; ${ }^{11}$ Division of Rheumatology, Hospital for Sick Children, Department of Pediatrics University of Toronto; ${ }^{12}$ Division of Rheumatology, Department of Medicine, University of Manitoba; ${ }^{13}$ General Internal Medicine, McGill University Health Centre, McGill University; ${ }^{14}$ Cochrane Musculoskeletal Group, University of Ottawa; ${ }^{15}$ Division of Rheumatology, Department of Medicine, Western University; ${ }^{16}$ Canadian Agency for Drugs and Technologies in Health; ${ }^{17}$ Department of Health Research Methods, Evidence and Impact, McMaster University

\subsection{6/lupus-2018-Ism.71}

Background To develop GRADE-based recommendations for the diagnosis and monitoring of systemic lupus erythematosus patients in Canada.

Methods Recommendations were developed using the GRADE (Grading of Recommendations, Assessment, Development and Evaluation) approach. The Canadian SLE Working Group (panel of Canadian rheumatologists and patient representative
Abstract CS-36 Table 1 Summary of recommendations with strength of evidence for the diagnosis and monitoring of SLE in Canada

Recommendation

Strength of

Recommendation; Quality of

Evidence

We recommend that all adult and pediatric patients

suspected of SLE be referred to a lupus specialist, most strong recommendation;

often a rheumatologist, to confirm diagnosis and be involved in ongoing care

For adult and pediatric patients with SLE, we suggest assessing disease activity with a validated instrument of disease activity during baseline and follow up visits. For adult and pediatric patients with SLE, we suggest assessing disease activity with a validated instrument of disease activity during baseline and follow up visits For adult lupus patients, we recommend that indicators of obesity, smoking status, diabetes, blood pressure and a basic lipid profile be measured upon diagnosis of SLE and be reassessed periodically according to current recommendations in the general population and be used to inform the cardiovascular risk assessment.

For adult patients with SLE, we suggest assessing the risk of osteoporosis and fractures every 1 to 3 years using a detailed history and focused physical examination, and measuring bone mineral density in patients with other risk factors according to recommendations in the general population. For all adults with SLE, we suggest screening 25hydroxyvitamin $D$ as part of the assessment for risk of osteoporosis and fractures

For adults and paediatric patients with a diagnosis of SLE and high risk behaviours for HCV acquisition, we recommend screening for Hepatitis $\mathrm{C}$ and repeating according to recommendations in the general population. For all other adult and paediatric patients with a diagnosis of SLE we suggest screening for Hepatitis $C$ and repeating according to recommendations in the general population

For adult patients with SLE who do not have clinical symptoms suggestive of osteonecrosis, we suggest not screening for or performing investigations for osteonecrosis. For patients who have suspected clinical symptoms of osteonecrosis, we suggest radiographs as the initial imaging modality rather than MRI or bone scan with SPECT according to recommendations in the general population.

For women with SLE, we suggest that anti-Ro and antiLa antibodies be measured immediately prior to pregnancy or during the first trimester.

For pregnant women with SLE, we suggest that uterine and umbilical Doppler studies be performed in the second or third trimester, or at the time of a suspected flare.

For women with prior or active lupus nephritis who are pregnant, we suggest measuring serum creatinine and urine protein to creatinine ratio every 4-6 weeks, or more frequently if clinically indicated. We suggest blood pressure and urinalysis be measured prior to pregnancy and every 4-6 weeks until 28 weeks, every 1-2 weeks until 36 weeks and then weekly until delivery. moderate quality evidence

conditional recommendation, low quality evidence

conditional recommendation, low quality evidence strong recommendation, moderate quality evidence conditional recommendation, low quality evidence moderate quality evidence conditional recommendation, low quality evidence

conditional recommendation, low quality evidence

conditional recommendation, low quality evidence

conditional recommendation; low quality evidence

conditional recommendation; low quality evidence strong recommendation, 


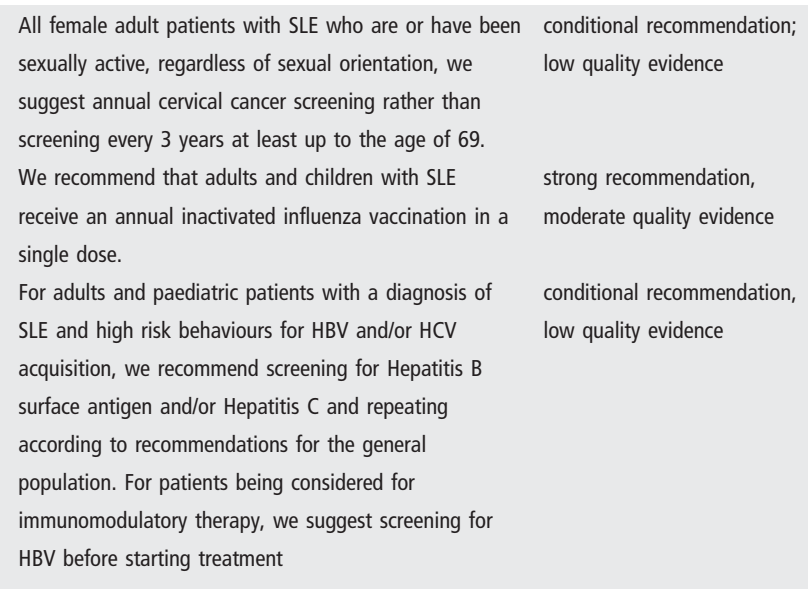

from CAPA (Canadian Arthritis Patient Alliance)) was created. A series of questions for recommendation development were identified based on the results of a survey of SLE practice patterns of members of the Canadian Rheumatology Association (CRA). Systematic literature reviews of randomized controlled trials and observational studies were conducted. Evidence to Recommendation Tables were prepared and presented to the panel at two face-to-face meetings for discussion and voting during and post-meeting online.

Results There were a total of fourteen recommendations for assessing and monitoring lupus patients (table 1). Three recommendations focused on disease activity and damage assessment suggesting that a validated disease activity score per visit and annual damage score were important in evaluating the patient. One strong recommendation was made for cardiovascular risk assessment with conditional recommendations for osteoporosis (2) and osteonecrosis (1). Three conditional recommendations were made regarding peripartum assessments, one on cervical cancer screening and two on hepatitis B and C screening. A strong recommendation was made for annual influenza vaccination.

Conclusions These are the first GRADE-based recommendations for the diagnosis and monitoring of SLE internationally. Evidence is largely of moderate to low quality resulting in more conditional versus strong recommendations. Further studies of higher quality and special attention to pediatric lupus populations are needed.

Acknowledgements The authors are members of the Canadian SLE Working Group which was created to develop these recommendations.

\section{CS-37 PREVALENCE OF COGNITIVE IMPAIRMENT IN SYSTEMIC LUPUS ERYTHEMATOSUS ASSESSED BY A COMPREHENSIVE NEUROPSYCHOLOGICAL BATTERY}

'Zahi Touma*, ${ }^{2}$ Robin Green, ${ }^{3}$ Lisa Engel, ${ }^{4}$ Carmela Tartaglia, ${ }^{2}$ Lesley Ruttan, ${ }^{2}$ Sabrina Lombardi, ${ }^{3}$ Nicole Anderson, ${ }^{3}$ Jiandong Su, ${ }^{3}$ Kenneth Colosimo, ${ }^{3}$ Michelle Vitti, ${ }^{3}$ Dennise Bonilla, ${ }^{3}$ Joan Wither, ${ }^{5}$ Marvin Fritzler, ${ }^{6}$ Dorcas Beaton. ${ }^{1}$ University of Toronto, Division of Rheumatology, Institute of Health Policy, Management and Evaluation, Toronto Western Hospital; ${ }^{2}$ Toronto Rehabilitation Institute; ${ }^{3}$ University of Toronto, Toronto Western Hospital; ${ }^{4}$ University of Toronto, Krembil Neurosciences Centre; ${ }^{5}$ University of Calgary; ${ }^{6}$ St Michael's Hospital

\subsection{6/lupus-2018-Ism.72}

Background Cognitive impairment (CI) is a common neurobehavioural manifestation of systemic lupus erythematosus (SLE). In our recent systematic review, the prevalence of CI was $38 \%$ (95\% CI $33 \%$ to $43 \%$ ) with a wide variation (15\%79\%), which may be due to differences in CI definitions and selection of neuropsychological tests across studies. We aim to report the prevalence of CI in a large cohort using a comprehensive battery $(\mathrm{CB})$ of tests in which we operationalized the classification of CI.

Methods Consecutive consenting SLE patients, aged 18-65 years, who attended a single center (Jul 2016-Feb 2018) were recruited. Patients were administered a CB that evaluates the major cognitive domains: Manual motor speed and dexterity, simple attention and processing speed, visual-spatial construction, verbal fluency, learning and memory (visuospatial and memory), executive functioning (untimed and timed).

Patient scores were compared to a normative sample of age- and gender-matched healthy controls to obtain z-scores. CI was operationalized on the $\mathrm{CB}$ as a $\mathrm{z}$-score of $\leq-1.5$ (as compared to controls) on $\geq 2$ domains or $\mathrm{z} \leq-2.0$ on $\geq 1$ domain. Descriptive statistics were used.

Results Of the 199 patients (89\% female), the mean age at SLE diagnosis was $28.3 \pm 10.4$ and disease duration at enrolment was $14.3 \pm 10.2$ years. The prevalence of CI was $37.7 \%$ ( $\mathrm{z} \leq-1.5$ in $\geq 2$ domains) and $49.8 \%$ ( $\mathrm{z} \leq-2.0$ in $\geq 1$ domains).

Prevalence of patients with domain $z$-scores of $\leq-1.5$ and $\leq-2.0$ varied from $3.0 \%-46.2 \%$ and $0.5 \%-25.1 \%$ respectively (figure 1). The most affected domain was learning and memory (visuospatial and memory) in 92 (46.2\%) patients based on $\mathrm{z} \leq-1.5$ on $\geq 2$ subtests and $50(25.1 \%)$ patients based on $\mathrm{z} \leq-2.0$ in $\geq 1$ subtest.

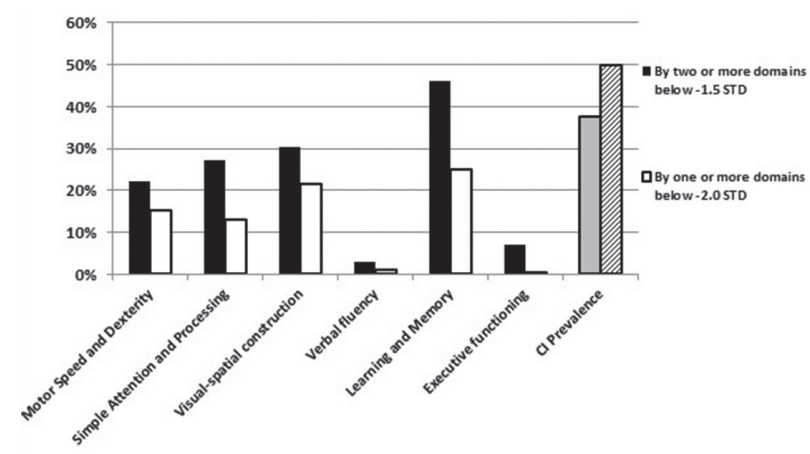

Abstract CS-37 Figure 1 Prevalence of patients with domain Z-scores of $\leq-1.5$ and $\leq 2.0$

Conclusion Prevalence of CI using our CB ranged between $37.7 \%-49.8 \% \quad(z \leq-1.5 \quad$ in $\geq 2 \quad$ domains and $z \leq-2.0 \quad$ in $\geq 1$ domains respectively), which was higher than the pooled prevalence from previous reports of $38 \%$. These differences in CI prevalence across studies could be attributed to different factors including the heterogeneity in patients' demographics/ comorbidities, sample size, the use of different metrics to determine CI, and the lack of a standardized definition of CI. Further studies are required to identify the best definition for CI and its metrics.

Acknowledgment This project is funded by the Arthritis Society and the Physicians' Services Incorporated Foundation. 